\title{
Immunohistochemical Localization of Periostin in Developing Long Bones of Mice
}

\author{
Yasushi Hirose ${ }^{1}$, Hironobu Suzuki ${ }^{1,2}$, Norio Amizuka', Junko Shimomura ${ }^{3}$, Yoshiro Kawano', Kayoko Nozawa- \\ INOUE ${ }^{1}$, Akira Kudo ${ }^{4}$ and Takeyasu MAEDA ${ }^{1}$ \\ 'Divisions of Oral Anatomy and ${ }^{2}$ Periodontology, Department of Oral Biological Science, ${ }^{3}$ Division of Pediatric Dentistry, Department of \\ Oral Health Science, Niigata University Graduate School of Medical and Dental Sciences, Niigata, Japan, ${ }^{4}$ Department of Life Science, \\ Faculty of Biological and Biotechnology, Tokyo Institute of Technology, Yokohama, Japan
}

(Received 29 January 2003; and accepted 31 January 2003)

\begin{abstract}
The immunolocalization of periostin, previously termed osteoblast-specific factor 2 , was investigated in developing long bones of 17-day-old fetal mice and of 1-, 2-, 3- and 8-week-old mice at the light and electron microscopic levels. Fetal femurs showed immunoreactions for periostin in the periosteum, perichondrium, articular surface of the epiphyseal cartilage, joint ligaments, and fascias of surrounding muscles. In particular, intense immunoreactivity for periostin was found in the fibrous layer of the periosteum and perichondrium. At postnatal 1-and 2-weeks, in contrast, the immunoreactivity was restricted to the periosteum and thick fascias of surrounding muscles when compared with the fetal bones. Immunoelectron microscopic observation of the periosteum demonstrated immunoreaction products for periostin at the junction of periosteal fibroblasts and collagen bundles, suggesting its competence in the cell-to-matrix interaction. Mice at 3 and 8 weeks, unlike 2-week-old mice, showed a periostin-immunoreaction dominantly in the osteoblastic layer but not in the fibroblastic layer of the periosteum. Furthermore, the perichondrium and fascias of surrounding muscles were devoid of immunoreaction. Thus, periostin was confirmed to be widely distributed in bone and concomitant tissues at the fetal stage. As mice grew, however, its immunoreactivity gradually came to be restricted to the osteoblastic layer of the periosteum. Our findings suggest that periostin acts at the site of the cell-to-matrix interaction in periosteum, fascias, and joint ligament during morphogenesis of these tissues.
\end{abstract}

Periostin, originally reported as osteoblast-specific factor 2 by Takeshita et al. (23), is a disulfate linked $90 \mathrm{kDa}$ protein secreted by osteoblasts. This protein has been isolated in previous studies aimed at identifying novel genes expressed specifically in the osteoblasts. Mouse periostin consists of 811 amino acids,

Address all correspondence: Takeyasu Maeda, DDS, PhD, Division of Oral Anatomy Department of Oral Biological Science Niigata University Graduate School of Medical and Dental Sciences 2-5274 Gakkocho-dori Niigata 951-8514, Japan

Tel: +81-25-227-2815 Fax: +81-25-223-6499

E-mail: maedat@dent.niigata-u.ac.jp and a computer analysis of its amino acid sequence has revealed an assumed complex protein structure with four repeats of a characteristic domain. The amino acid sequence of periostin has a high homology with Fasciclin I that is expressed in the central nervous system of insects, serving in neural cell-tocell contact $(1,24)$. Therefore, periostin has been regarded as a member of Fasciclin I family including Big-h3 (21), Algal-CAM (7), and midline fasciclin (6), mimicking a function of Fasciclin I. Unlike Fasciclin I, however, periostin is also distributed abundantly in the periosteum and periodontal ligament as condensed extracellular components (5). Typically, the extracellular matrix plays a pivotal role in main- 
taining local homeostasis and histological architectures for a variety of tissues. Many extracellular matrix-associated proteins - connective tissue growth factor (15), nephroblastoma overexpressed (NOV) (17), vitronectin and fibronectin - may regulate cellular activities such as cell adhesion, migration, mitogenesis, differentiation, survival, angiogenesis, wound healing and tumorgenesis (15). Periostin may interact with these extracellular matrices in the periosteum and periodontal ligaments.

Horiuchi et al. (5) first reported the localization and expression of this novel protein, osteoblast-specific factor 2 , in the periosteum and periodontal ligament of adult mice, and renamed this protein as "periostin" to avoid any confusion with a transcription factor that used this acronym, also known as Cbfal (2). However, recent studies have confirmed the presence of periostin in non-small cell lung carcinoma (18), thyroma (19), the embryonic mouse heart (13), the neonatal rat kidney (10), neuroblastoma (20) and tumor-derived ovarian epithelial cells (8). Therefore, periostin should be regarded as a fetal oncoprotein that is involved in the morphogenesis and subsequent development of various tissues, including bone.

In this study, we have undertaken an examination of the spatio-temporal localization of periostin in developing mouse femurs and surrounding tissues by immunocytochemical technique to provide a cue for verifying biological functions of this protein.

\section{MATERIALS AND METHODS}

The care and use of animals followed "The Guiding Principles for the Care and Use of Animals", approved by Niigata University in accordance with the principles of the declaration of Helsinki.

Tissue preparation. Under anesthesia with an intraperitoneal injection of chloral hydrate $(400 \mathrm{mg} / 100 \mathrm{~g}$ body weight), 1-, 2-, 3- and 8-week-old SPF/VAF mice and fetal mice in their 17th day of gestation were perfused through the left ventricle either with $4 \%$ paraformaldehyde in a $0.1 \mathrm{M}$ phosphate buffer $(\mathrm{pH} \mathrm{7.4)}$, or with a mixture of $4 \%$ paraformaldehyde and $0.0125 \%$ glutaraldehyde in a $0.067 \mathrm{M}$ phosphate buffer for light microscopic or immunoelectron microscopic observation, respectively. Femurs with surrounding muscular tissues were immediately extracted and immersed in the same fixatives for additional $8 \mathrm{~h}$ at $4{ }^{\circ} \mathrm{C}$. The specimens were decalcified with $10 \%$ EDTA-2 $\mathrm{Na}$ solution at $4{ }^{\circ} \mathrm{C}$ and equilibrated in a $30 \%$ sucrose solution overnight for cryo- protection. Cryostat sections were prepared and mounted onto glass slides pre-coated with 3-aminopropyltriethoxy silane.

Immunohistochemistry for periostin. Cryostat sections were processed for the avidin-biotin-complex (ABC) method. After the blocking of endogenous peroxidase activity with methanol containing $0.3 \%$ $\mathrm{H}_{2} \mathrm{O}_{2}$ for $30 \mathrm{~min}$, they were incubated with a rabbit polyclonal antiserum to periostin (5) at a dilution of $1: 100$ for $2-3 \mathrm{~h}$ at room temperature. Sections were then incubated with biotinylated goat anti-rabbit IgG (1 : 1000; Vector lab. Inc., Burlingame, CA) and subsequently with an avidin-peroxidase complex (Vector) for $60 \mathrm{~min}$ each at room temperature. The antigen-antibody reaction sites were made visible by incubation with $0.04 \% 3-3^{3}$ diaminobenzidine and $0.03 \%$ hydrogen peroxide. Immunostained sections were counter-stained with $0.03 \%$ methyl green, dehydrated through an ascending series of ethanol, and cover-slipped with Permount (Fisher Scientific, Springfield, NJ).

For immunoelectron microscopy, immunostained cryostat sections were post-fixed with $1 \%$ osmium tetraoxide reduced with $1.5 \%$ potassium ferrocyanide in a $0.1 \mathrm{M}$ cacodylate buffer for $3 \mathrm{~h}$ at $4^{\circ} \mathrm{C}$, and were dehydrated in ascending acetones prior to embedding in epoxy resin (Epon 812, Taab, Berkshire, UK). Ultrathin sections were prepared with an ultramicrotome, and examined with a Hitachi H-7000 transmission electron microscope (Hitachi Co. Ltd., Tokyo, Japan), following brief staining with uranyl acetate and lead citrate.

\section{RESULTS}

Immunolocalization of periostin in femurs of 17-day. old fetal mice

Fetal femurs at 17 days of gestation showed a broad and exuberant distribution of the immunoreaction for periostin in the perichondrium and periosteum (Fig. 1a). This immunoreaction was clearly detected in the fibrous layers of the periosteum and perichondrium, which are composed of fibroblastic cells that interconnect surrounding muscles and the inner osteoblastic layer of the periosteum/perichondrium (Fig. 1c, 1d). In contrast, the osteoblastic layer of the periosteum, which consists of mature osteoblasts and preosteoblasts, displayed a weak immunoreactivity for periostin. Unlike periosteum, the endosteum covering the metaphyseal trabecular bones and the diaphyseal endosteum facing the bone marrow region did not show any immunoreactivity for peri- 


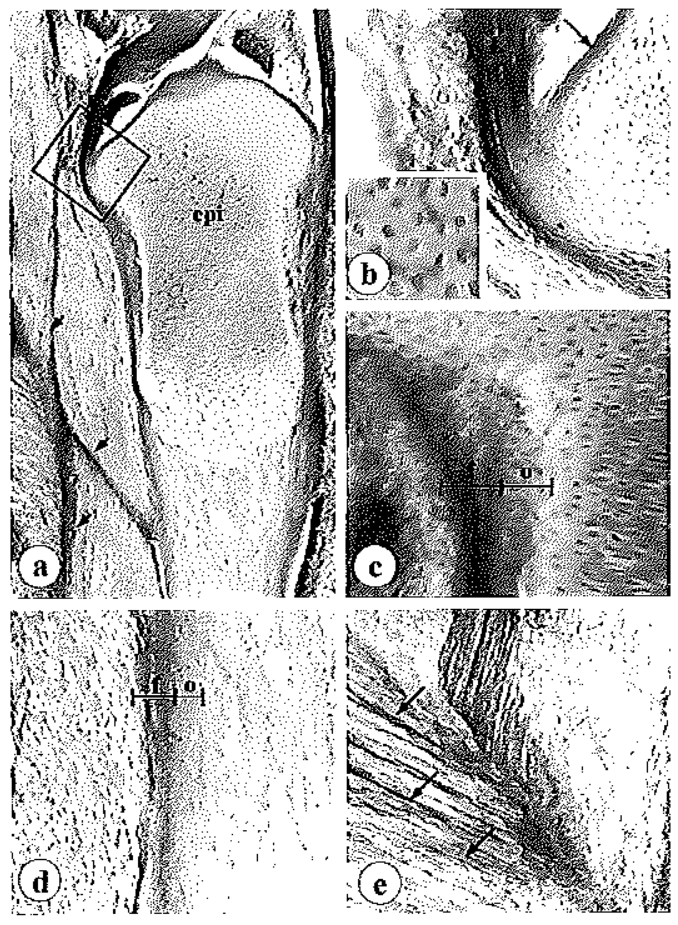

Fig. I Immunolocalization of periostin in a 17-day-old fetal femur. (a) The broad distribution of periostin (deep black) is seen in the fetal femur and surrounding muscular and fascias (arrows) tissues. epi: epiphysis (b) Periostin is immunolocalized intensely in the ligamentous tissues and superficial layer of the articular surface, and faintly in the epiphyseal chondrocytes (inset). (c, d) The fibrous layers ( $\mathrm{f}$ in $\mathrm{c}$ and $\mathrm{d}$ ) of the perichondrium and periosteum show a strong immunopositivity for periostin but a weak reactivity occurs in the osteoblastic layers (o). Note the absence of any immunoreaction in the region of the endosteum. (e) Fascias of surrounding muscles are positive for periostin immunohistochemistry. Fine linear structures (arrows) identical to the endomysium are immunopositive as well. Original magnification: $\mathrm{a}, \times 30, \mathrm{~b}, \times 100, \mathrm{c}, \mathrm{d}, \times 150, \mathrm{e}, \times 120$

ostin. The ligamentous tissues of a knee joint and the articular surface of the epiphyseal cartilage were also positive in periostin-immunoreaction (Fig. 1b). A faint immunoreaction was recognizable in chondrocytes in the reservoir and proliferative zones of the epiphyseal cartilage. The fascias - including the epimysium and perimysium of peripheral skeletal muscles - demonstrated a strong immunopositivity for periostin (Fig. le). Thus, in the fetal stage, periostin was distributed ubiquitously in periosteum, perichondrium, fascias of muscles, and ligamentous tissues.
Immunolocalization of periostin in femtrs of 1 -weekold mice

In 1-week-old postnatal mice, the perichondrium adjacent to the hypertrophic zone of the femoral epiphyseal cartilage was positive for periostin, while the perichondrium encapsulating the epiphyseal extremity was negative in immunoreaction (Fig. 2a, 2c). The superficial layer of the epiphyseal articular surface exhibited a weak immunopositivity. In contrast, immunohistochemistry visualized a definite periostin-immunoreactivity in knee-joint ligaments (Fig. 2b) and the fibrous layer of the femoral perios-

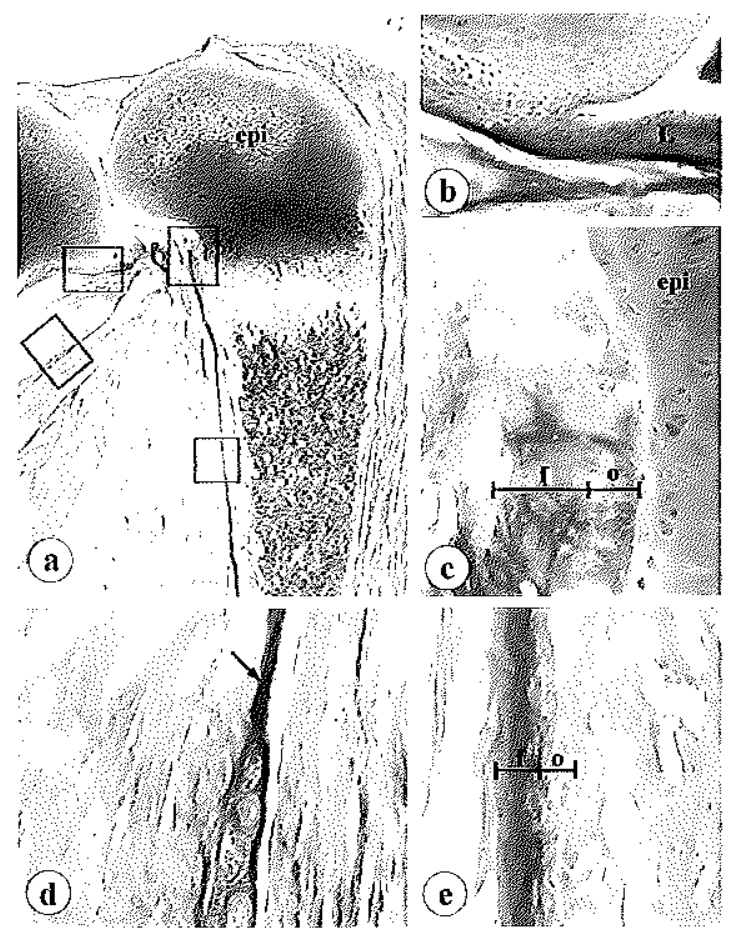

Fig. 2 Immunolocalization of periostin in a 1-weekold postnatal femur. (a) At 1 week of age, periostin is restricted to the skeletal tissues relative to the fetal femur. Fig. b-e are higher magnifications of boxed areas in Fig. a. epi: epiphysis (b) A joint ligamant is intensely positive for periostin. (c) The fibrous layer of the perichondrium adjacent to the hypertrophic zone of the epiphyseal cartilage displays positivity for periostin. In contrast, the upper region of the perichondrium close to the epiphyseal extremity is less immunoreactive. (d) The thick fascias (perimysium) of surrounding muscles exhibit periostin, whereas less immunoreactivity is seen in the region of the endomysium. (e) The fibrous layer (f) of the periosteum, compared with the osteoblastic layer (o), reveals a strong immunopositivity. Note the absence of any immunoreaction in the endosteal osteoblasts. Original magnification: $a, \times 20, b, \times 80, c, \times$ $200, \mathrm{~d}, \times 80, \mathrm{c}, \times 150$ 
teum (Fig. 2e). Thick fascias corresponding to the perimysium of surrounding muscles expressed a significant periostin-immunoreactivity, whereas the endomysium lacked this (Fig. 2d). Thus, the broad distribution of periostin shown at the fetal stage was markedly reduced at 1 week after birth, resulting in a restricted localization in the femurs and concomitant tissues.

Immunoelectron microscopy demonstrated that immunoreaction products for periostin were confined to the cytoplasmic processes of fibroblasts in the periosteal fibrous layer (Fig. 3). However, these immunoreactions were not evenly distributed along the entire surface of the fibroblastic cells; they were prominent on cell membranes of those cytoplasmic processes tightly associated with the bundles of collagen fibers. A faint and dispersed immunoreactivity was seen in the collagen bundles which were juxtaposed with the immunopositive cytoplasmic processes.

Immunolocalization of periostin in femurs of 2-, 3and 8-week-old mice

Examination of 2-, 3- and 8-week-old mice showed selective immunopositivity in the periosteum in all stages (Figs. 4a, 5a and 6a). The predominant immunoreactivity was found in the periosteal fibrous layer at 2 weeks (Fig. 4b, 4d), while the osteoblastic

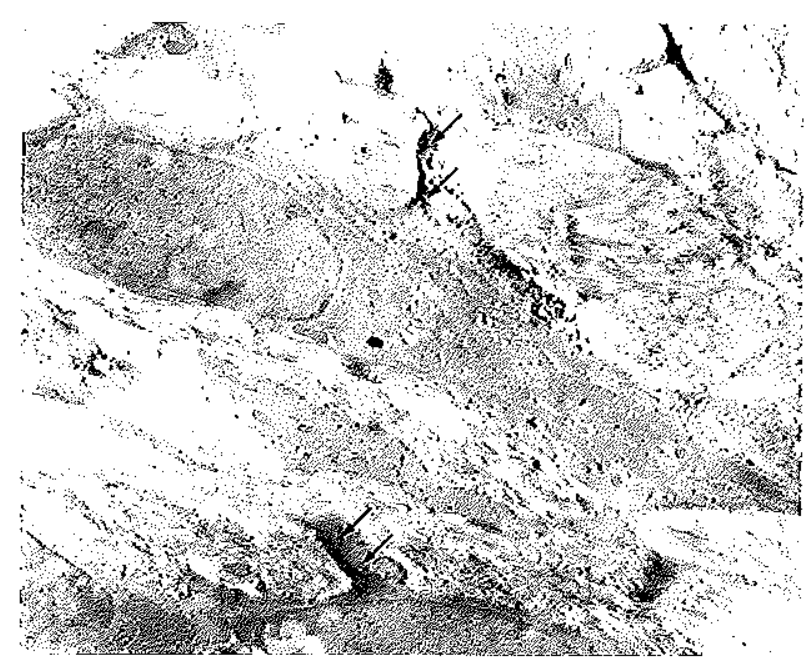

Fig. 3 Immunoelectron microscopic image of periostin. Periostin immunopositivity (black color) is seen on cytoplasmic processes (arrows) of fibroblastic cells in the periosteal fibrous layer. Note that the immunopositivity does not appear evenly on the entire surface of the fibroblastic cells. Collagen bundles close to the immunopositive cytoplasmic process are faintly positive for periostin as well. Original magnification: $\times 3000$

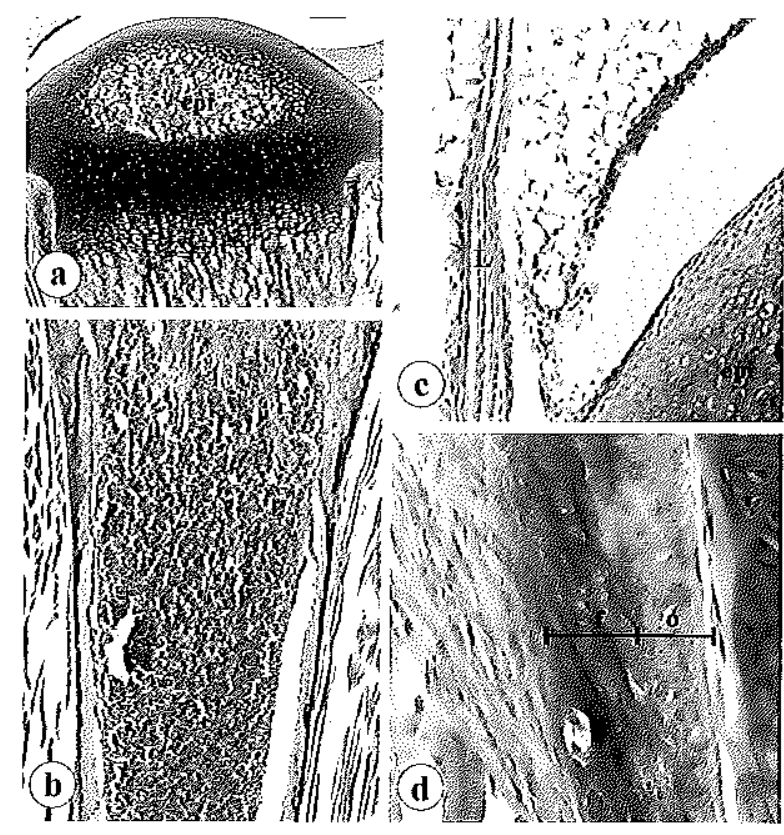

Fig. 4 Immunolocalization of periostin in the femur of a 2-week-old mouse. (a) Perichondrium and periosteum surrounding the epiphyseal cartilage show the periostinimmunoreactivity. epi: epiphysis (b) In the diaphysis, the periostin immunoreactivity is seen in the region of the periosteum, whereas no immunopositivity is apparent in the region of the endosteum. (c) A joint ligament (L) and the articular surface of the epiphysis are weakly immunopositive for periostin. (d) When examined at a higher magnification, the fibrous layer of the perichondrium exhibits intense periostin inmmunoreaction. Original magnification: $a, b, \times 20, c, \times 80, d, \times 150$

layer - but not the fibrous layer of the periosteum - exhibited a periostin-immunoreaction in mice at 3 weeks of age (Fig. 5c). Ligaments of knee joints of both 2-and 3-week-old mice were faintly periostin-positive (Fig. 4c, 5b). However, fascias of surrounding muscles were devoid of any periostinimmunoreaction. Femurs of 8-week-old adult mice showed a distinct immunoreactive osteoblastic layer of the periosteum (Fig. 6b). The endosteum, perichondrium, and fascias of surrounding muscles did not show any specific immunoreaction.

\section{DISCUSSION}

The present immunohistochemical study was able to demonstrate clearly the spatio-temporal localization of periostin in developing femurs and concomitant tissues. As a consequence, a broad distribution of a periostin-immunoreaction was found in fetal skeletal tissues including the periosteum, perichondrium, fas- 
cias of surrounding muscles, and ligamentous tissues. However, as the mice matured, this protein came to show a localization restricted to the perios-

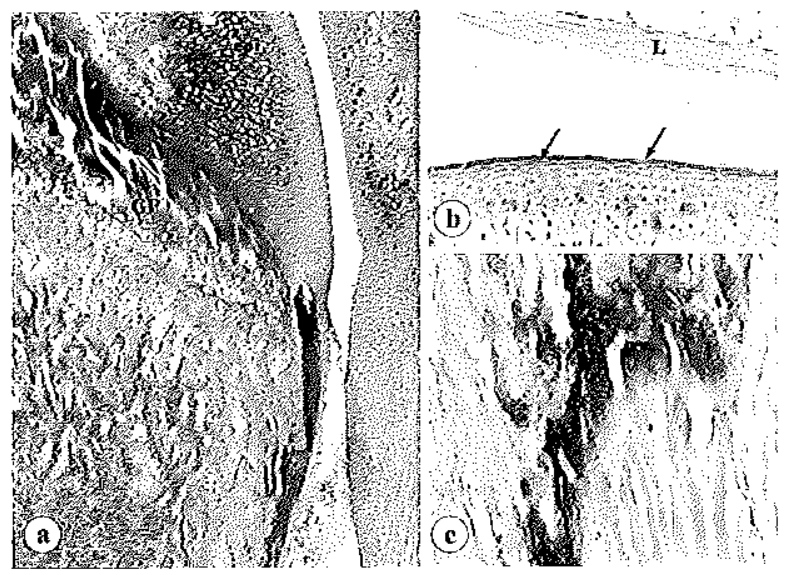

Fig. 5 Immunolocalization of periostin in the femur of a 3-week-old mouse. (a) A femur at 3 weeks of age displays an immunopositive periostcum. epi: epiphysis, GP: growth plate (b) The superficial layer of the epiphyseal articular surface is positive for periostin (arrows), while the ligament (L) of knee joints is faintly stained for the periostin immunoreactivity. (c) In contrast, fascias of surrounding muscles are negative for the immunoreactivity. The periosteum of a 3-week-old mouse reveals distinct periostin immunoreactivity mainly in the osteoblastic layer rather than the fibrous layer.Original magnification: $\mathrm{a}, \times 40, \mathrm{~b}, \times 80, \mathrm{c}, \times 80$

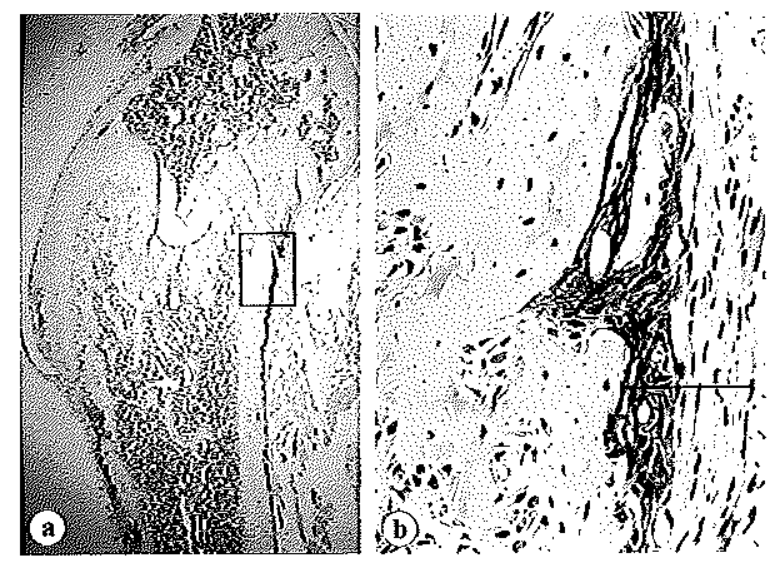

Fig. 6 Immunolocalization of periostin in the femur of an 8-week-old mouse. (a) Low powered image of the 8-week-old femur display periostin obviously restricted to the periosteum. (b) When observed at a higher magnification, the immunopositive osteoblastic layer is distinguishable from the fibrous layer. Note the absence of any immunoreactive osteoblasts in the endosteum. Original magnification: $a, \times 10, b, \times 150$ teum as seen in the 8 -week-old mice. The prevalent localization of periostin in the fetal bone suggested its pivotal role in skeletal tissue formation. Despite the initial assumption of its possessing a property termed the "osteoblast- specific factor", endosteal osteoblasts in vivo did not express a discernible amount of this protein. Recent studies have revealed undetectable transcripts of periostin in the osteocytic cell line (12), but an intense and broad expression of periostin in various tissues and cells including nonosteocytic tumor cell lines $(8,10,13,18-20)$. These findings imply a more generalized function of this protein. For instance, periostin was expressed in the developing heart from the 10.5-day-old embryonic stage to an especially high level in the newly born in cardiac mesenchymal cells, but not within either the developing myocardial cells or endothelial cells. We found abundant periostin-immunoreaction in fascias including the endomysium and perimysium of peripheral skeletal muscles. It is likely that periostin in the endomysium is involved in the cellular interaction between myoblasts and mesenchymal cells of the endomysium, and this protein regulates the differentiation of normal muscle cells as previously suggested (9). Thus, periostin is produced as a kind of fetal oncoprotein, and the biological function of periostin might be ubiquitous for the morphogenesis and subsequent development of various tissues.

The precise role of periostin has remained veiled, despite its implicative localization in skeletal tissues and others. Periostin has been postulated to modulate cell-to-matrix interaction (5). This conjecture is based on its predominant localization in dense connective tissues such as the periosteum, which is subjected to the physical force of exercise. Coincidently, we confirmed its long-lasting localization at both fetal and adult stages. Unlike the periosteum, the endosteum is a thin cell layer composed of mature osteoblasts and preosteoblasts loosely connected with a smaller amount of intercellular matrices. The cell-to-matrix interaction of the endosteum, therefore, does not appear physiologically equivalent to that of the periosteum. Thus, the attachments between periosteal fibroblasts or osteoblasts and thick bundles of collagen fibers can serve as adhesive equipments bearing mechanical stress, giving rise to the strength and rigidity of this tissue. Electron microscopic observation manifested the ultrastructural localization of periostin on the cell membranes of fibroblastic cells with which collagen bundles were tightly associated. Thus, periostin situates itself at a tight association-site between cells and extracellular components in a dense connective 
tissue, and might behave as a kind of adhesion molecule, as previously proposed by an analysis of its amino acid sequence (23).

It is likely that periostin is an adhesion molecule: a distinct membrane surface receptor that partakes in coordinating vital biological events such as morphogenesis, cell migration, and intercellular communication. Because of a high homology in amino acid sequences, periostin has been considered a Fasciclin I family that is expressed in the central nervous system of insects, acting on neuronal cell-to-cell adhesion, cell aggregation, and cell sorting $(1,24)$. Therefore, periostin may be capable of mimicking a function as Fascilcin I. Horiuchi et al. (5) postulated that periostin supports cell spreading and the attachment of MC3T3-E1 cells, judging from the observation that anti-periostin antibodies impeded MC3T3E1 cell spreading on periostin-coated plates employing a solid phase binding assay. Our immunohistochemistry confirmed periostin in the periosteum, fascias, and ligamentous tissues in which cells are spreading interstitially among luxuriant extracellular collagen bundles, and therefore corroborates his notion. In addition, as shown in the electron microscopy, thick collagen bundles of periosteum appear to serve as scaffolds for fibroblastic attachment by intermediating periostin between collagen bundles and fibroblastic cells.

Integrins, a family of transmembrane glycoproteins consisting of noncovalent heterodimers, interact with a wide variety of ligands such as extracellular matrices and their associating proteins. Gillan et al. (4) reported that periostin secreted by epithelial ovarian carcinoma is a ligand for $\alpha v \beta 3$ and $\alpha \mathrm{v} \beta 5$ integrins and promotes cell motility, though periostin does not contain an RGD motif. Since periostin is abundantly expressed in the periosteum and periodontal ligaments (5), integrins expressed in these tissues may interact with periostin. Fukumoto et al. (3) demonstrated that normal rabbit periosteum expressed $\alpha$ I, III, $\mathrm{V}$ and $\beta 1$ subunits of integrins at low levels in the periosteum. Meanwhile, $\alpha v \beta 3$ and $\alpha \mathrm{v} \beta 1$ integrins are rich in the periodontal ligament; the former's ligand is vitronectin, the latter's ligands are fibronectin and collagenous cementum attachment protein $(11,22)$. Taken together, these facts indicate that periostin might recognize the $\alpha_{\mathrm{V}}$ integrin subunit in the periosteum, and modulate a cell-to-matrix interaction by mediating this integrin subunit.

There is still on-going discussion as to why periostin comes to be highly restricted to the periosteum with aging, though it is earlier widespread in various fetal tissues. Periostin may be intrinsic for the morphogenesis of ligaments, periosteum, and muscles including fascias rather than for maintenance of the histological architecture of these tissues. Our immunoelectron microscopy showed the possibility of periostin acting at the site of the cell-to-matrix interaction. Extracellular matrices of the periosteum and periodontal ligament are highly remodeled in accordance with mechanical stress due to exercise and occlusion. These facts show that periostin may modulate the remodeling and metabolism of extracellular matrices by mediating a cell-to-matrix interaction, and could be widely distributed in various fetal tissues in which extracellular matrices are actively remodeled. In contrast, joint ligaments, the epimysium, and perimysium in adult mice do not seem to show a high turnover of the remodeling of extracellular matrices. Alternatively, it is possible that periostin inhibits precocious cell differentiation to keep its proliferative potentiality in the periosteal fibrous layer, joint ligaments, and articular surfaces of the epiphyseal cartilage at the fetal stage. A recent study has discovered that periostin is a target for Twist, a basic helix-loop-helix transcription factor (16). This transcriptional factor binds to the Twist box located on the mouse periostin promoter, and simulates the transcriptional activity of periostin. Twist transcripts and periostin are co-localized in the region of the osteogenic front of calvarial sutures of newborn mice, in which many undifferentiated osteogenic cells are pooled. All this points to the conclusion that the negative regulation of Twist for osteoblastic differentiation (14) could be mediated by periostin.

In this study, we demonstrated the detailed immunolocalization of periostin in the periosteum, joint ligamentous tissues, and articular surface at the fetal stage. However, it was gradually limited to the periosteum when the mice aged. Electron microscopy verified periostin situated at the contact site between cytoplasmic process and extracellular collagen bundles, therefore indicating that periostin modulates a cell-to-matrix interaction. However, the relevant role of periostin is still under conjecture. Further investigations will be necessary for elucidating the biological function of periostin.

\section{Acknowledgements}

The authors thank Mr. M. Hoshino and Mr. K. Takeuchi for their technical assistance. This work is supported by Grants-in Aid from the Ministry of Education, Science and Culture, Japan (Norio Ami- 
zuka, Takeyasu Maeda).

\section{REFERENCES}

1. Bastiani M. J., Harrelson A. L., Snow P. M. and Goodman C. S. (1987) Expression of fasciclin I and II glycoproteins on subsets of axon pathways during neuronal development in the grasshopper. Cell 48, 745-755.

2. Ducy P., Zhang R., Geoffroy V., Ridall A. L. and Karsenty G. (1997) Osf2/Cbfa1: a transcriptional activator of ostcoblast differentiation. Cell 89, 747-754.

3. Fukumoto T., Sanyal A., Fitzsimmons J. S. and O'Driscoll S. W. (2002) Expression of betal integrins during periosteal chondrogenesis. Osteoarthritis Cart. 10, 135-144.

4. Gillan L., Matei D., Fishman D. A., Gerbin C. S., Karlan B. Y. and Chang D. D. (2002) Periostin secreted by epithelial ovarian carcinoma is a ligand for alpha(V)beta(3) and alpha(V)beta(5) integrins and promotes cell motility. Cancer Res. 62, 5358-5364.

5. Horiuchi K., Amizuka N., Takeshita S., Takamatsu H., Katsuura M., Ozawa H., Toyama Y., Bonewald L. F. and Kudo A. (1999) Identification and characterization of a novel protein, periostin, with restricted expression to periosteum and periodontal ligament and increased expression by transforming growth factor beta. J. Bone Miner: Res. 14, 1239-1249.

6. Hu S., Somnenfeld M., Stahl S., Crews S. T. (1998) Midline Fasciclin: a Drosophila Fasciclin-I-related membrane protein localized to the CNS midline cells and trachea. $J$. Neurobiol. 35, $77-93$.

7. Huber O. and Sumper M. (1994) Algal-CAMs: isoforms of a cell adhesion molecule in embryos of the alga Volvox with homology to Drosophila fasciclin I. EMBO J. 13, 4212-4222.

8. Ismail R. S., Baldwin R. L., Fang J., Browning D., Karlan B. Y., Gasson J. C. and Chang D. D. (2000) Differential gene expression between nomal and tumor-derived ovarian epithelial cells. Cancer Res. 60, 6744-6749.

9. Itoh M., Tsukada S., Orita T., Nishiu J., Tomoike H., Nakainura Y. and Tanaka T. (1998) Identification by differential display of eight known genes induced during in vivo intimal hyperplasia. J. Hum. Genet. 43, 9-13.

10. Ito T., Struki A., Imai E., Horimoto N., Ohnishi T., Daikuhara Y. and Hori M. (2002) Tornado extraction: a method to enrich and purify RNA from the nephrogenic zone of the neonatal rat kidney. Kidney, Int. 62, 763-769.

11. Ivanovski S., Komaki M., Bartold P. M., Narayanan A. S. (1999) Periodontal-derived cells attach to cementum attachment protein via alpha 5 beta 1 integrin. J. Periodontal Res. 34, $154-159$.

12. Kato Y., Windle J. J., Koop B. A., Mundy G.R. and Bonewald L. F. (1997) Establishment of an osteocyte-like cell line,
MLO-Y4 J. Bone Miner: Res. 12, 2014-2023.

13. KruzynskamFrejtag A., Machnicki M., Rogers R., Markwald R. R., Conway S. J. (2001) Periostin (an osteoblast-specific factor) is expressed within the embryonic mouse heart during valve formation. Mech. Dev. 103, 183-188.

14. Lee M. S., Lowe G. N., Strong D. D., Wergedal J. E. and Glackin C. A. (1999) TVIST, a basic helix-loop-helix transcription factor, can regulate the human osteogenic lineage. $J$. Cell Biochem. 75, 566-577.

15. Moussad, E. and Brigstock DR. (2000) Connective tissue growth factor: What's in a name? Mol. Genet. Metab. 71, 276-292.

16. Oshima A., Tanabe H., Yan T., Lowe G. N., Glackin C. A. and Kudo A. (2002) A novel mechanism for the regulation of osteoblast differentiation: transcription of periostin, a member of the fasciclin I family, is regulated by the bHLH transcription factor, twist. J. Cell Biochem. 86, 792-804.

17. Perbal B. (2001) NOV (nephroblastoma overexpressed) and the CCN family of genes: structural and functional issues. Mol. Pathol. 54, 57-79.

18. Sasaki H., Lo K. M., Chen L. B., Auclair D., Nakashima Y., Moriyama S., Fukai I., Tam C., Loda M. and Fujii Y. (2001) Expression of Periostin, homologous with an insect cell adhesion molecule, as a prognostic marker in non-small cell lung cancers. Jpn. J. Cancer Res. 92, 869-873.

19. Sasaki H., Auclair D., Kaji M., Fukai L., Kiriyama M., Yamakawa Y., Fujii Y. and Chen L. B. (2001) Serum level of the periostin, a homologue of an insect cell adhesion molecule, in thymoma patients. Cancer Lett. 172, 37-42.

20. Sasaki H., Sato Y., Kondo S., Fukai I., Kiriyama M., Yamakawa Y. and Fuji Y. (2002) Expression of the periostin mRNA level in neuroblastoma. J. Pediatr: Surg. 37, 12931297.

21. Skonier J., Neubauer M., Madisen L., Bennett K., Plowman G D. and Purchio A. F. (1992) cDNA cloning and sequence analysis of beta ig-h3, a novel gene induced in a human adenocarcinoma cell line after treatment with transforming growth factor-beta. DNA Cell Biol. 11, 511-422.

22. Steffensen B., Duong A. H., Milam S. B., Potempa C. L., Winborn W. B., Magnuson V. L., Chen D., Zardeneta G. and Klebe R. J. (1992) Immunohistological localization of cell adhesion proteins and integrins in the periodontium. $J$. Periodontol. 63, 584-592.

23. Takeshita S., Kikuno R., Tezuka K. and Amann E. (1993) Osteoblast-specific factor 2: cloning of a putative bone adhesion protein with homology with the insect protein fasciclin I. Biochem. J. 294, 271-278.

24. Zinn K., McAllister L. and Goodman C. S. (1988) Sequence analysis and neuronal expression of saciclin $l$ in grasshopper and Drosophila. Cell 53, 577-587. 